\title{
Epidemiological and imaging features that can affect the detection of ureterolithiasis on ultrasound
}

\author{
Epidemiologia e achados por imagem que podem interferir na detecção da ureterolitíase \\ na ultrassonografia
}

Daniela Rebouças Nery ${ }^{1}$, Yves Boher Costa ${ }^{2}$, Thais Caldara Mussi ${ }^{3}$, Ronaldo Hueb Baroni ${ }^{3}$

Nery DR, Costa YB, Mussi TC, Baroni RH. Epidemiological and imaging features that can affect the detection of ureterolithiasis on ultrasound. Radiol Bras. 2018 Set/Out;51(5):287-292.

Abstract Objective: To identify, in patients with clinical suspicion of ureterolithiasis, epidemiological and imaging features that affect calculus detection on ultrasound, as well as to compare ultrasound with multidetector computed tomography (MDCT).

Materials and Methods: We searched our database for patients who underwent ultrasound, followed by MDCT (if the ultrasound was negative), for suspected ureterolithiasis in an emergency setting. Patients were divided into three groups: positive ultrasound (US+); negative ultrasound/positive MDCT (US-/MDCT+); and negative ultrasound/negative MDCT (US-/MDCT-). We evaluated age, gender, ureterolithiasis laterality, location of the calculus within the ureter, body mass index, calculus diameter, and calculus attenuation on MDCT.

Results: Of a total of 292 cases of suspected ureterolithiasis, 155 (53.1\%) were in the US+ group, 46 (15.7\%) were in the US-/ MDCT+ group, and 91 (31.2\%) were in the US-/MDCT- group. There were no significant differences among the groups in terms of age, gender, ureterolithiasis laterality, and mean MDCT attenuation values. Distal ureterolithiasis was most common in the US+ group, and calculi at other ureteral locations were more common in the US-/MDCT+ group. The mean body mass index was significantly higher in the US-/MDCT+ group than in the US+ group, and the mean calculus diameter was significantly greater in the US+ group than in the US-/MDCT+ group.

Conclusion: A high body mass index, large calculus diameter, and calculus location in the distal third of the ureter are the major factors favoring ureterolithiasis detection on ultrasound.

Keywords: Ureterolithiasis; Ultrasonography; Tomography, X-ray computed; Body mass index.

Resu mo Objetivo: Identificar achados epidemiológicos e de imagem em pacientes com suspeita clínica de ureterolitíase que afetam a detecção do cálculo no ultrassom (US), comparado com a tomografia computadorizada multidetectores (TCMD).

Materiais e Métodos: Procuramos, em nosso banco de dados, por pacientes que realizaram US no serviço de emergência, seguido por TCMD (se US negativo), por suspeita de ureterolitíase. Os pacientes foram divididos em: 1) US positivo; 2) US negativo e TCMD positiva; 3) US e TCMD negativos. Avaliamos idade, sexo, lateralidade, localização ureteral, índice de massa corporal, diâmetro e densidade do cálculo.

Resultados: Foram incluídos no estudo 292 pacientes. Constatamos que 53,1\% das ureterolitíases foram detectadas por US (grupo 1), 15,7\% apenas por TCMD (grupo 2), e em 31,2\% ambos os métodos foram negativos (grupo 3). Idade, sexo, lateralidade e densidade do cálculo pela TCMD não tiveram diferença significativa entre os grupos. No grupo 1, ureterolitíase foi detectada, preferencialmente, em localização distal, e fora do ureter distal no grupo 2. 0 índice de massa corporal foi significativamente maior em pacientes do grupo 2, comparado ao grupo 1 . 0 diâmetro do cálculo detectado no grupo 1 foi significativamente maior do que no grupo 2. Conclusão: Índice de massa corporal, diâmetro e localização do cálculo no terço distal do ureter foram os principais fatores que contribuíram para a detecção do cálculo pelo US.

Unitermos: Ureterolitíase; Ultrassonografia; Tomografia computadorizada; Índice de massa corporal.

\section{INTRODUCTION}

Ureterolithiasis causing acute flank pain is a common clinical situation in the emergency room $^{(1-3)}$. Ultrasound

Study conducted at the Hospital Israelita Albert Einstein, São Paulo, SP, Brazil.

1. Hospital Universitário Professor Edgard Santos - Universidade Federal da Bahia (UFBA) e Delfin Medicina Diagnóstica, Salvador, BA, Brazil,

2. MedRadius, Maceió, AL, Brazil,

3. Hospital Israelita Albert Einstein, São Paulo, SP, Brazil.

Correspondence: Dra. Thais Caldara Mussi. Hospital Israelita Albert Einstein. Avenida Albert Einstein, 627, Jardim Leonor. São Paulo, SP, Brazil, 05652-900. E-mail: thaiscaldara@gmail.com. is used extensively in the examination of patients with suspected ureterolithiasis and has the advantage of being universally available, fast, and easily performed, as well as not employing ionizing radiation ${ }^{(1)}$.

Ureterolithiasis can be accompanied by dilatation of the renal collecting system, depending on the size/location of the calculus, the duration of the resulting obstruction, and whether that obstruction is partial or complete ${ }^{(4)}$. Factors such as calculus size, patient weight, and body mass

Received July 6, 2017. Accepted after revision August 23, 2017. 
index (BMI) can influence ureterolithiasis detection by ultrasound $^{(5)}$. In addition, the thickness of subcutaneous fat and its sound-attenuating properties reduce the accuracy of ultrasound ${ }^{(6)}$. Furthermore, the sensitivity of ultrasound depends on calculus size and location ${ }^{(7)}$. Fowler et al. ${ }^{(8)}$ found that ultrasound is a poor modality for identifying renal calculi smaller than $0.4 \mathrm{~cm}$ in diameter. Compared with computed tomography (CT), ultrasound has lower accuracy in the detection of ureterolithiasis, with a reported sensitivity and specificity of $11-93 \%$ and $95-100 \%$, respectively ${ }^{(5)}$.

For a number of years, unenhanced multidetector CT (MDCT) has been the gold standard for diagnosing urinary tract calculi in adults ${ }^{(3,9,10)}$. The method reportedly has a sensitivity and specificity higher than $95 \%$ in detecting ureterolithiasis $^{(11,12)}$. In addition, MDCT is suitable for detecting abnormalities that are unrelated to ureteral calculi. However, MDCT involves the use of radiation ${ }^{(13)}$, is more costly than ultrasound ${ }^{(14)}$, and, in rare cases, can present pitfalls in interpretation, such as mistaking a phlebolith for a distal ureterolithiasis ${ }^{(15)}$.

Given the higher cost of MDCT compared with ultrasound, together with the fact that a number of patients submitted to ultrasound will also need to undergo MDCT if the ultrasound findings are inconclusive ${ }^{(16)}$, one way to avoid diagnostic delays and to reduce costs would be to determine which patients would benefit from undergoing MDCT initially. It should also be borne in mind that, in most cases, ureterolithiasis is characterized by acute flank pain. Therefore, any attempt to expedite the diagnosis should be encouraged.

To our knowledge, there have been no studies investigating the effects that epidemiologic and topographical factors, as well as BMI, have on the detection of ureterolithiasis with ultrasound and MDCT. Therefore, the aim of the present study was to evaluate the epidemiological and imaging features that can affect the detection of ureterolithiasis on ultrasound.

\section{MATERIALS AND METHODS}

\section{Study sample}

This was a retrospective study of data related to the imaging examinations of patients who underwent ultrasound of the urinary tract in the emergency room, during a period of one year. We identified cases through searches of the database at our institution. We included cases in which the patient had positive ultrasound findings that explained the emergency room admission (ureterolithiasis or other condition requiring emergency treatment) or, if the ultrasound results were negative or inconclusive, had undergone MDCT within the first $24 \mathrm{~h}$ after the ultrasound. The exclusion criteria were as follows: having negative ultrasound findings and not having undergone MDCT to confirm the absence of disease; no epidemiological information being available; and images being unavailable. The local institutional review board approved the study and, because it was a retrospective study, waived the requirement for informed consent.

Of the 1630 patients who underwent ultrasound of the urinary tract during the study period, 1338 were excluded: because the ultrasound findings were negative and the patient did not undergo MDCT ( $\mathrm{n}=1299)$; because there was no epidemiological information available $(\mathrm{n}=38)$; or because there were no images available $(n=1)$. Therefore, the final sample comprised 292 patients, who were divided into three groups (Figure 1): positive ultrasound (US+); negative ultrasound/positive MDCT (US-/MDCT+); and negative ultrasound/negative MDCT (US-/MDCT-).

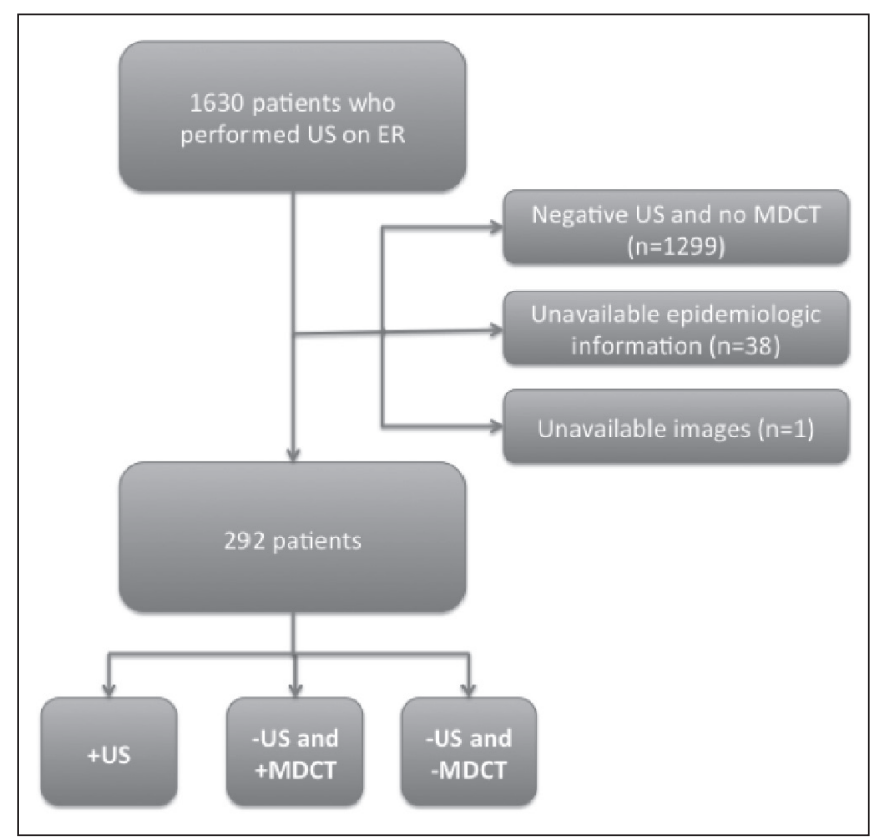

Figure 1. Flow chart of inclusion and exclusion criteria.

Epidemiological and topographical factors were compared among the groups. The parameters analyzed were the age, gender, and BMI of the patient, together with the laterality, size, location, and attenuation of the ureterolithiasis. The location of the calculus in the ureter was classified as follows (Figure 2): the ureteropelvic junction (UPJ); the proximal ureter (portion of the ureter that extends from the UPJ to the upper border of the sacroiliac joint); the mid-ureter (between the upper and lower borders of the sacroiliac joint); the distal ureter (portion of the ureter that extends from lower border of the sacroiliac joint to the ureterovesicular junction [UVJ]); and the UVJ. For the patients who had undergone both types of examinations, MDCT was considered the reference.

\section{Imaging}

Ultrasound examinations were performed with one of two different ultrasound systems (HDI 5000 or IU22; Philips Medical Systems, Amsterdam, the Netherlands). Curved phased-array $(3.5-5.0 \mathrm{MHz})$ transducers were used. All examinations were conducted by radiologists with at least four 


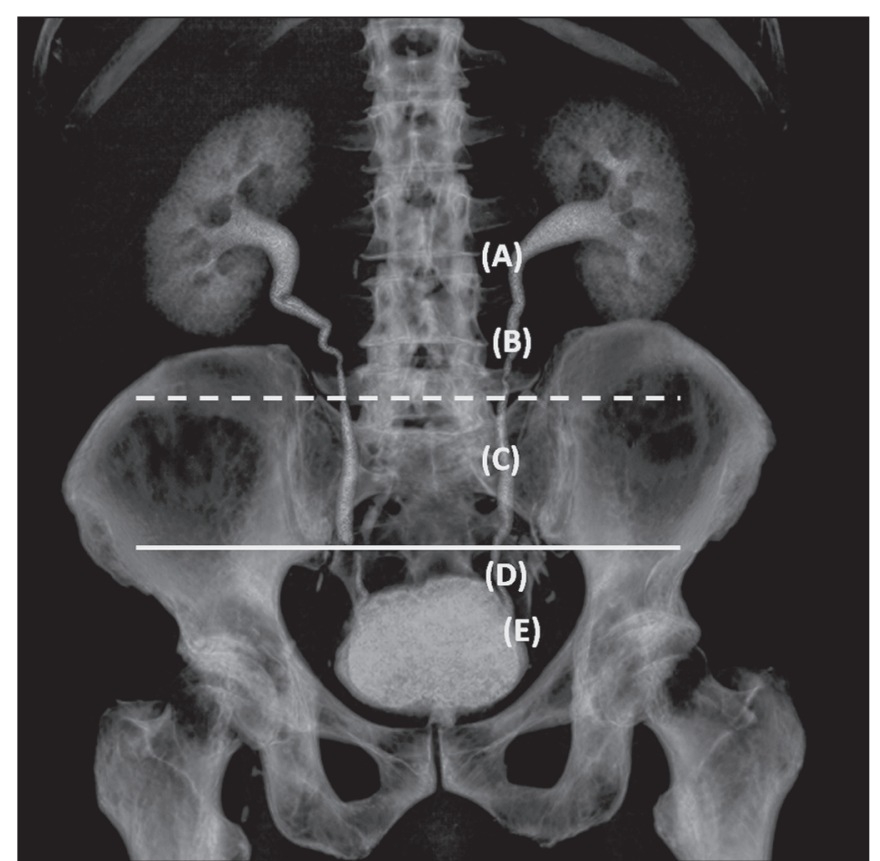

Figure 2. Three-dimensional reconstruction of an MDCT examination of the urinary tract. Note the ureteral anatomic division: the UPJ (A); the proximal ureter (B); the mid-ureter (C), between the upper border of sacroiliac joint (dashed line) and its lower border (solid line); the distal ureter (D); and the UVJ (E).

years of experience in evaluating abdominal ultrasounds. Ureteral calculi were measured at their longest diameter.

MDCT examinations were performed in 64- or 320-detector row scanners (Aquilion 64 or Aquilion One; Toshiba Medical Systems, Tokyo, Japan). In accordance with the protocol of our institution, there was no oral or intravenous administration of contrast medium. MDCT images extended from the upper poles of the kidneys through the pubic symphysis. A board-certified radiologist, blinded to the ultrasound results, reviewed the MDCT examinations, measured the calculus size (on its longitudinal and perpendicular axis), and determined its attenuation (in HU).

\section{Statistical analysis}

Statistical analyses were performed with the Statistical Package for the Social Sciences, version 17.0 (SPSS Inc., Chicago, IL, USA) and with the program R (R Development Core Team, 2012). Univariate analysis was performed with chi-square or Fisher's exact tests to analyze each categorical variable (gender, ureterolithiasis laterality, and calculus location). One-way analysis of variance was performed to analyze continuous variables (age, BMI, calculus size, and calculus density). In a multivariate analysis, we performed stepwise logistic regression to identify factors that predicted the detection of ureterolithiasis by ultrasound. The analyses were performed with the Statistical Analysis System software, version 9.3 (SAS Institute Inc., Cary, NC, USA). Receiver operating characteristic (ROC) curve analysis was performed with the Number Cruncher Statistical System program, version 2004 (NCSS, Kaysville, UT, USA). For all analyses, values of $p<0.05$ were considered statistically significant.

\section{RESULTS}

Ultrasound was performed in all 292 patients in the sample (168 men, 124 women; mean age, 47.7 years). Of those 292 patients, $155(53.1 \%)$ were in the US+ group, 46 (15.7\%) were in the US-/MDCT+ group, and 91 (31.2\%) were in the US-/MDCT- group.

\section{Ureterolithiasis laterality}

Of the 155 calculi identified by ultrasound, 85 (54.8\%) were in the left ureter and $70(45.2 \%)$ were in the right ureter. Of the 46 calculi identified only by MDCT, 26 (56.5\%) were in the left ureter and $20(43.5 \%)$ in the right ureter. The laterality of the calculi did not differ significantly different between the US+ and US-/MDCT+ groups $(p=0.954)$.

\section{Calculus location}

Of the 155 calculi identified by ultrasound, $12(7.8 \%)$ were in the UPJ, $17(11.0 \%)$ were in the proximal ureter, $14(9.1 \%)$ were in the mid-ureter, $63(40.9 \%)$ were in the distal ureter, and $48(31.2 \%)$ were in the UVJ. Of the 46 calculi identified only by MDCT, $1(2.2 \%)$ was in the UPJ, $10(21.7 \%)$ were in the proximal ureter, $10(21.7 \%)$ were in the mid-ureter, $16(34.7 \%)$ were in the distal ureter, and 9 (19.5\%) were in the UVJ. The proportion of patients with calculi in distal locations (the distal ureter or UVJ) was highest in the US+ group $(p=0.036)$.

\section{BMI}

The mean BMI was $25 \mathrm{~kg} / \mathrm{m}^{2}$ in the US+ group, $27 \mathrm{~kg} /$ $\mathrm{m}^{2}$ in the US-/MDCT+ group, and $24 \mathrm{~kg} / \mathrm{m}^{2}$ in the US-/ MDCT- group, the difference between the US-/MDCT+ group and the two other groups being statistically significant $(p=0.028)$. Figure 3 illustrates the case of a patient with a BMI of $39 \mathrm{~kg} / \mathrm{m}^{2}$, in whom ultrasound failed to detect the ureterolithiasis, which was diagnosed on the basis of the MDCT findings (US-/MDCT+ group).

\section{Calculus diameter and MDCT attenuation}

When determined by ultrasound, the mean diameter of the calculi was $0.62 \pm 0.3 \mathrm{~cm}$ (range, $0.1-1.6 \mathrm{~cm}$ ), compared with $0.46 \pm 0.2 \mathrm{~cm}$ (range, $0.2-1.0 \mathrm{~cm}$ ) when it was determined by MDCT (in the transverse plane). The mean diameter of the calculi was greater in the US+ group than in the US-/MDCT+ group $(0.62 \mathrm{~cm}$ vs. $0.46 \mathrm{~cm})$, and the difference was significant $(p<0.001)$. The mean MDCT attenuation for the calculi was slightly higher in the US+/ MDCT+ group than in the US-/MDCT+ group (1117 \pm $349 \mathrm{HU}$ vs. $902 \pm 394 \mathrm{HU}$ ), although the difference was not statistically significant $(p=0.235)$.

\section{Epidemiological factors}

There were no statistical differences among the groups in terms of the mean age $(p=0.821)$ or the gender distribution $(p=0.589)$. 

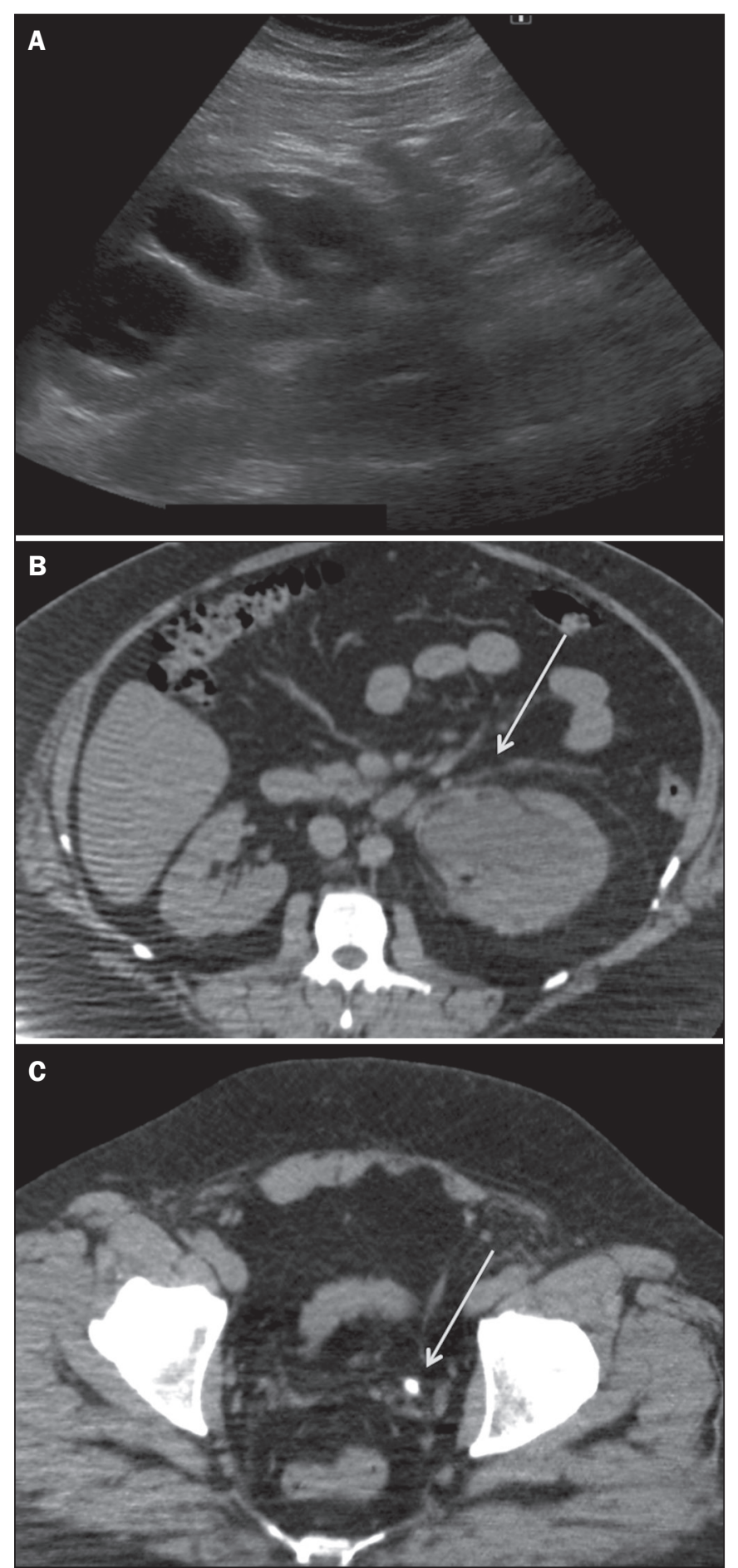

Figure 3. A 43-year-old male, $\mathrm{BMI}=39$, with abdominal pain. Ultrasound image (A) showing marked left collecting system dilatation without detecting the obstructive cause. Following ultrasound, MDCT was performed which confirmed the collecting system dilatation (B) and a calculus in the distal ureter $(\mathbf{C})$ (arrows), measuring $0.6 \mathrm{~cm}$.

\section{Differential diagnoses of acute abdominal pain}

Acute abdominal pain was attributed to a cause other than ureterolithiasis in $33(11.3 \%)$ of the 292 patients. Of those 33 patients, $20(60.6 \%)$ were female and $13(39.4 \%)$ were male. Two of those 33 patients were diagnosed with hemorrhagic ovarian cyst; six patients were diagnosed with pyelonephritis; 14 patients were diagnosed with pyeloureteritis cystica; and one patient each was diagnosed with acute prostatitis, diverticulitis, omental infarction, focal pyelonephritis, bladder calculi, nephrocalcinosis, polycystic kidney with bleeding, lumbar vertebral fracture, ureteral stenosis, and urothelial tumor.

\section{Multivariate analyses and ultrasound false-negative results}

Using logistic regression (Table 1), we found that a BMI $\leq 27 \mathrm{~kg} / \mathrm{m}^{2}$, a calculus diameter (in the transverse plane on MDCT) of $0.5-0.7 \mathrm{~cm}$, and location of the calculus in the distal ureter were the best predictors of ultrasound detection, with respective correlation coefficients of 1.15 (vs. $>27 \mathrm{~kg} / \mathrm{m}^{2}$ ), 0.61 (vs. $<0.5 \mathrm{~cm}$ ), and 0.08 (vs. a proximal location). Figure 4 illustrates the case of a patient in which a calculus with a diameter of $0.5 \mathrm{~cm}$, located in the mid-ureter, was detected by ultrasound (US+ group).

For each unit increase in BMI, we observed an increase of $16 \%$ in likelihood of false-negative ultrasound results. In addition, the likelihood of a false-negative ultrasound result increased $234 \%$ for calculi with a diameter $<0.5 \mathrm{~cm}$ than for those with a diameter of $0.5-0.7 \mathrm{~cm}$, whereas increased $426 \%$ if the calculus was located in the proximal ureter than if it was located in the distal ureter. For each millimeter increase in the size of the calculus, the likelihood of false negative of ureterolithiasis by ultrasound decreased $40 \%(p<0.001)$.

\section{Determination of the optimal BMI cut-off value for ultrasound detection of ureterolithiasis}

Using a ROC curve analysis (Figure 5), we found that, for optimal detection of ureterolithiasis by ultrasound, patients should have a BMI $\leq 27 \mathrm{~kg} / \mathrm{m}^{2}(p=0.005)$. That cutoff value was found to have a sensitivity, specificity, positive predictive value, and negative predictive value of $63.0 \%$, $61.3 \%, 33.0 \%$ and $95.0 \%$, respectively, for the ultrasound detection of ureterolithiasis.

Table 1-Multivariate logistic regression analysis of factors potentially pre dictive of false-negative ultrasound results for ureterolithiasis $(n=201)$.

\begin{tabular}{lcc}
\hline Independent variables & P-value & OR $(95 \% \mathrm{Cl})$ \\
\hline Age (each year) & 0.821 & $1(0.97-1.04)$ \\
BMI (each unit) & $0.007 *$ & $5(1.04-1.28)$ \\
Ureterolithiasis laterality (left vs. right) & 0.785 & $1.12(0.5-2.48)$ \\
Gender (female vs. male) & 0.589 & $1.28(0.53-3.08)$ \\
Calculus diameter & & \\
$\quad$ (< 0.5 cm vs. 0.5-0.7 cm) & $<0.001 *$ & $3.54(1.53-8.21)$ \\
$\quad$ (> 0.7 cm vs. 0.5-0.7 cm) & $0.002 *$ & $0.19(0.04-0.8)$ \\
Calculus location & & \\
$\quad$ (UPJ vs. distal) & 0.663 & $1.04(0.11-10.24)$ \\
$\quad$ (Proximal vs. distal) & $0.018 *$ & $4.56(1.47-14.18)$ \\
$\quad$ (Mid-ureter vs. distal) & 0.058 & $3.75(1.24-11.34)$ \\
$\quad$ (UVJ vs. distal) & $0.011 *$ & $0.5(0.19-1.35)$ \\
\hline
\end{tabular}

OR, odds ratio; $\mathrm{Cl}$, confidence interval. 

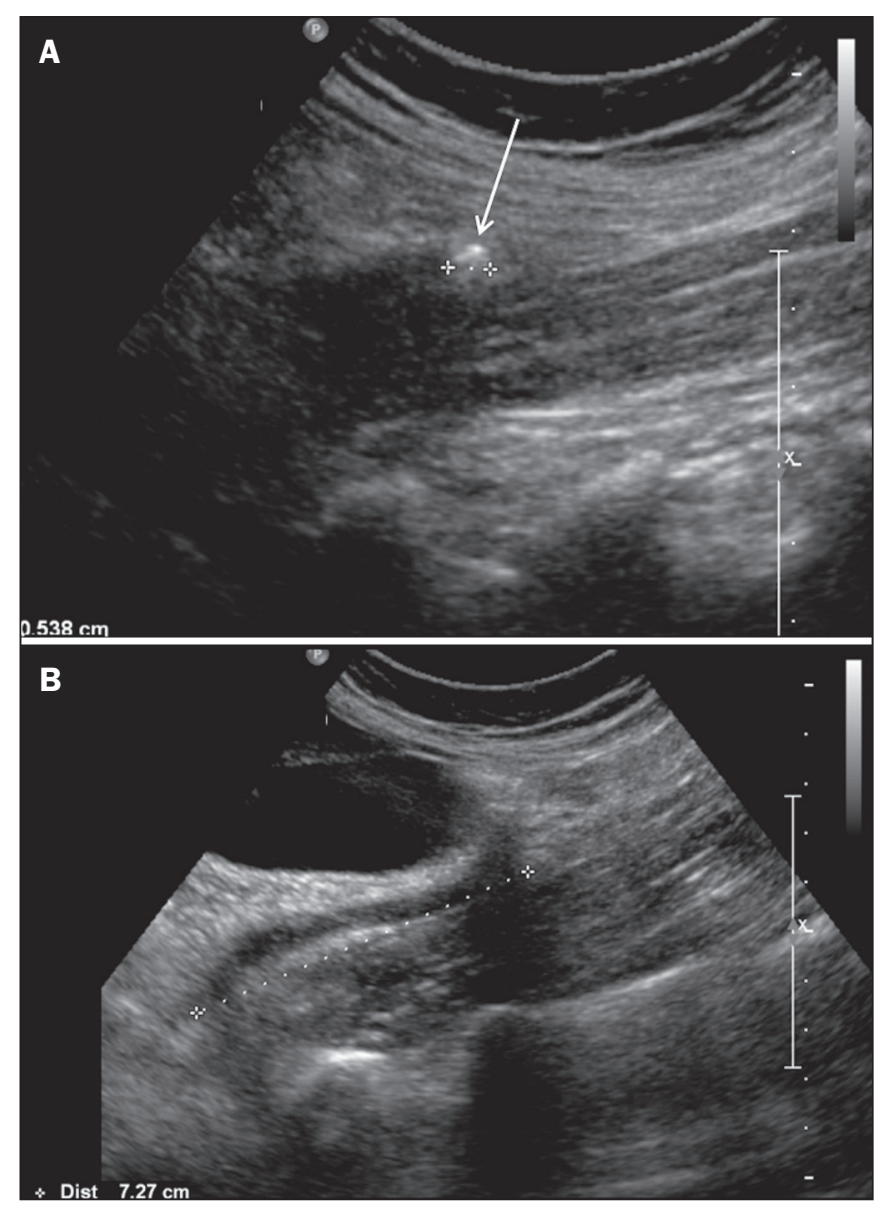

Figure 4. A 47-year-old female with a BMI of $22 \mathrm{~kg} / \mathrm{m}^{2}$ and ureteral colic. UItrasound showing a calculus measuring $0.5 \mathrm{~cm}$ (arrow) in the mid-ureter (A), $7.0 \mathrm{~cm}$ below the renal pelvis (B).

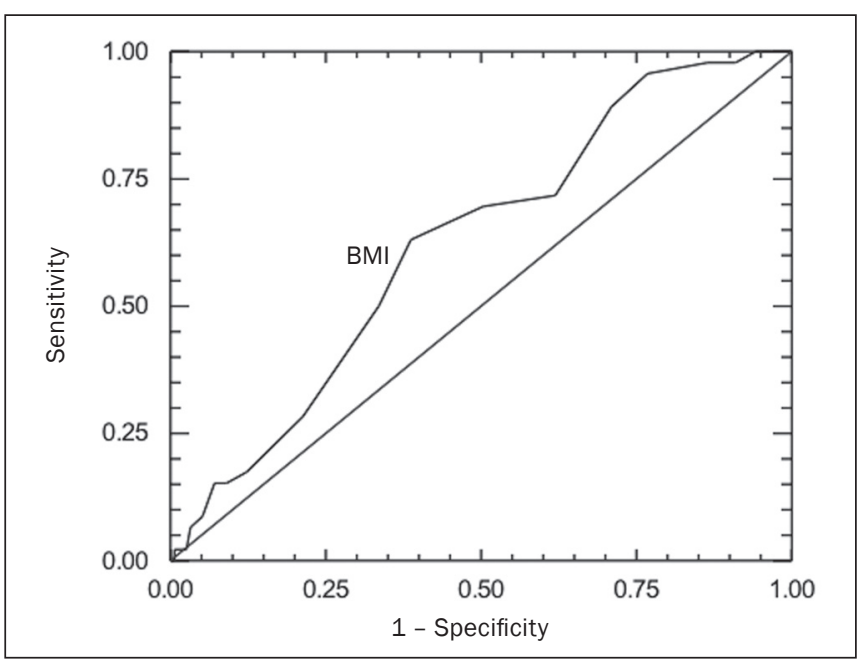

Figure 5. ROC curve analysis for ultrasound detection of ureterolithiasis, according to BMI.

\section{DISCUSSION}

Choosing the correct initial imaging modality is an essential part of the management of cases in which the patient presents to the emergency department with flank pain. Various imaging modalities are available to evaluate hydronephrosis and renal calculi (conventional X-ray of the abdomen; specific X-ray examination of the kidney, ureter, or bladder; ultrasound; MDCT; and magnetic resonance imaging), although most recent protocols limit the choices of initial imaging modalities in an acute setting to MDCT and ultrasound ${ }^{(17,18)}$.

In the present study, we aimed to determine which epidemiological and imaging findings should be considered when selecting the first-line diagnostic tool for the identification of ureterolithiasis. In addition, we attempted to determine to what degree the size and location of a calculus in the ureter can affect the ability of ultrasound to detect it.

According to our findings, the use of ultrasound in patients with a BMI $>27 \mathrm{~kg} / \mathrm{m}^{2}$ can delay diagnosis and treatment because a greater number of patients will also need to undergo MDCT to detect the calculi. One previous report showed that protocols involving early CT can reduce the overall cost by shortening hospital stays ${ }^{(19)}$.

Previous studies have reported that MDCT has high sensitivity and specificity (97\% and 96\%, respectively) for the detection of ureterolithiasis ${ }^{(11,12)}$. For ultrasound, the reported sensitivity for the detection of ureterolithiasis varies widely, from $11 \%$ to $93 \%^{(5,7,14,20)}$. In addition, ultrasound can overestimate the calculus size, especially for calculi with a diameter $\leq 0.5 \mathrm{~cm}^{(5)}$. The accurate measurement of the diameter of a calculus and the determination of its location are essential to predicting the chances of its spontaneous passage and defining its correct management ${ }^{(21)}$.

Previous studies have considered a cut-off BMI of $>30 \mathrm{~kg} / \mathrm{m}^{2}$ to choose between low-dose and standard CT protocols ${ }^{(17,22)}$. However, to our knowledge, there have been no studies taking patient BMI into consideration when evaluating the efficacy of ultrasound for ureterolithiasis detection. According to our results, ultrasound is of limited value for diagnosing ureterolithiasis in patients with a BMI $>27 \mathrm{~kg} / \mathrm{m}^{2}$. In fact, in an obese patient with $8 \mathrm{~cm}$ of subcutaneous fat, $94 \%$ of the ultrasound wave is absorbed before it reaches the peritoneal cavity ${ }^{(23)}$. The effect of obesity on the accuracy of ultrasound in detecting ureterolithiasis has not been investigated in depth. Ulusan et al. ${ }^{(6)}$ found no correlation between BMI and the rate of detection of renal calculi with ultrasound, although their study involved only renal calculi and not ureteral calculi.

We found that most of the ureteral calculi identified on ultrasound were located in the distal third of the ureter, especially in the UVJ, which is similar to the findings of other studies $^{(1,13)}$. Distal locations (the distal ureter and UVJ) accounted for $72.1 \%$ of all such calculi. Other studies have also shown that the detection ability of ultrasound varies depending on the location of the calculus in the ureter ${ }^{(1,13)}$.

Ureteral calculi $<0.5 \mathrm{~cm}$ in diameter can be overlooked on ultrasound because they do not cast an acoustic shadow and may not be distinguishable from the normally hyperechoic renal sinus ${ }^{(6)}$. Previous research has demonstrated that the sensitivity of ultrasound for renal calculus 
detection is dependent on calculus size ${ }^{(8)}$. In the present study, we found significantly different detection rates for the different sizes of calculi.

Some epidemiological factors, such as gender, can be important to define the presence of ureterolithiasis. In the present study, the incidence of back pain was higher among premenopausal women than among men, although all such women were in the US-/MDCT- group (i.e., no ureterolithiasis was detected) and the difference was not statistically significant. That finding might be related to a discretely higher incidence of pyelonephritis among the females in the sample.

Ulusan et al. ${ }^{(6)}$ reported that, using ultrasound, it was more difficult to visualize small renal calculi in the left kidney than in the right kidney. However, we observed no significant difference between the right and left ureters in terms of the sensitivity of ultrasound for the detection of calculi.

Of the 292 patients in our sample, $33(11.3 \%)$ presented a diagnosis other than ureteral calculus. Similar incidences of alternative or additional causes to flank pain have previously been observed ${ }^{(24,25)}$, which supports the recommendation for additional investigation after an initial imaging examination excludes ureterolithiasis.

Our study has some limitations. It was a retrospective study of data obtained with different types of MDCT scanners and ultrasound machines, which might have introduced some variability into our results. In addition, a large number of patients were excluded because they had negative ultrasound results and did not undergo MDCT within the following $24 \mathrm{~h}$. Furthermore, a number of radiologists with different levels of experience analyzed the ultrasound images. However, at many facilities, ultrasound is performed by a radiology technician rather than a radiologist. Finally, we had no access to patient histories, which could be a limitation, because some patients could have had prior episodes of ureterolithiasis.

We conclude that, among the patient-related variables that can be measured prior to imaging, BMI is the only one that can be taken into consideration when trying to decide whether ultrasound or MDCT should be performed first. Because there is no way of knowing the location or size of the ureteral calculus beforehand, the findings that calculus size and location in the ureter affect ultrasound detection of ureterolithiasis have no clinical relevance.

\section{REFERENCES}

1. Mos C, Holt G, Iuhasz S, et al. The sensitivity of transabdominal ultrasound in the diagnosis of ureterolithiasis. Med Ultrason. 2010;12:188-97.

2. Hess B. Pathophysiology, diagnosis and conservative therapy in calcium kidney calculi. Ther Umsch. 2003;60:79-87.

3. Park SJ, Yi BH, Lee HK, et al. Evaluation of patients with suspected ureteral calculi using sonography as an initial diagnostic tool: how can we improve diagnostic accuracy? J Ultrasound Med. 2008;27:1441-50.
4. Kamaya A, Wong-You-Cheong J, Park HS, et al. Diagnostic ultrasound: abdomen and pelvis. Philadelphia, PA: Elsevier; 2015.

5. Ray AA, Ghiculete D, Pace KT, et al. Limitations to ultrasound in the detection and measurement of urinary tract calculi. Urology. 2010;76:295-300.

6. Ulusan S, Koc Z, Tokmak N. Accuracy of sonography for detecting renal stone: comparison with CT. J Clin Ultrasound. 2007;35:25661.

7. Nicolau C, Claudon M, Derchi LE, et al. Imaging patients with renal colic_consider ultrasound first. Insights Imaging. 2015;6:441-7.

8. Fowler KA, Locken JA, Duchesne JH, et al. US for detecting renal calculi with nonenhanced CT as a reference standard. Radiology. 2002;222:109-13.

9. Catalano O, Nunziata A, Altei F, et al. Suspected ureteral colic: primary helical CT versus selective helical CT after unenhanced radiography and sonography. AJR Am J Roentgenol. 2002;178:379-87.

10. Vrtiska TJ, Hattery RR, King BF, et al. Role of ultrasound in medical management of patients with renal stone disease. Urol Radiol. 1992;14:131-8.

11. Smith RC, Verga M, McCarthy S, et al. Diagnosis of acute flank pain: value of unenhanced helical CT. AJR Am J Roentgenol. 1996; 166:97-101.

12. Sourtzis S, Thibeau JF, Damry N, et al. Radiologic investigation of renal colic: unenhanced helical CT compared with excretory urography. AJR Am J Roentgenol. 1999;172:1491-4.

13. Patlas M, Farkas A, Fisher D, et al. Ultrasound vs CT for the detection of ureteric stones in patients with renal colic. Br J Radiol. 2001;74:901-4.

14. Ripollés T, Errando J, Agramunt M, et al. Ureteral colic: US versus CT. Abdom Imaging. 2004;29:263-6.

15. Traubici J, Neitlich JD, Smith RC. Distinguishing pelvic phleboliths from distal ureteral stones on routine unenhanced helical CT: is there a radiolucent center? AJR Am J Roentgenol. 1999;172:13-7.

16. Grisi G, Stacul F, Cuttin R, et al. Cost analysis of different protocols for imaging a patient with acute flank pain. Eur Radiol. 2000; 10:1620-7.

17. Brisbane W, Bailey MR, Sorensen MD. An overview of kidney stone imaging techniques. Nat Rev Urol. 2016;13:654-62.

18. Coursey CA, Casalino DD, Remer EM, et al. ACR Appropriateness Criteria ${ }^{\circledR}$ acute onset flank pain-suspicion of stone disease. Ultrasound Q. 2012;28:227-33.

19. Patel M, Han SS, Vaux K, et al. A protocol of early spiral computed tomography for the detection of stones in patients with renal colic has reduced the time to diagnosis and overall management costs. Aust N Z J Surg. 2000;70:39-42.

20. Sheafor DH, Hertzberg BS, Freed KS, et al. Nonenhanced helical CT and US in the emergency evaluation of patients with renal colic: prospective comparison. Radiology. 2000;217:792-7.

21. Jendeberg J, Geijer H, Alshamari M, et al. Size matters: the width and location of a ureteral stone accurately predict the chance of spontaneous passage. Eur Radiol. 2017;27:4775-85.

22. Fulgham PF, Assimos DG, Pearle MS, et al. Clinical effectiveness protocols for imaging in the management of ureteral calculous disease: AUA technology assessment. J Urol. 2013;189:1203-13.

23. Modica MJ, Kanal KM, Gunn ML. The obese emergency patient: imaging challenges and solutions. Radiographics. 2011;31:811-23.

24. Katz DS, Scheer M, Lumerman JH, et al. Alternative or additional diagnoses on unenhanced helical computed tomography for suspected renal colic: experience with 1000 consecutive examinations. Urology. 2000;56:53-7.

25. Ahmad NA, Ather MH, Rees J. Incidental diagnosis of diseases on un-enhanced helical computed tomography performed for ureteric colic. BMC Urol. 2003;17:2. 